\title{
Ultrasonic Periodontal Probing Based on the Dynamic Wavelet Fingerprint
}

\author{
Jidong Hou \\ Department of Applied Science, The College of William \& Mary, Williamsburg, VA 23187-8795, USA \\ Email: jbhou@wmalumni.com
}

S. Timothy Rose

Valley Periodontics, 2535 Northern Road, Appleton, WI 54914, USA

Email: roset@ada.org

Mark K. Hinders

Department of Applied Science, The College of William \& Mary, Williamsburg, VA 23187-8795, USA

Email: hinders@as.wm.edu

Received 17 August 2004; Revised 4 October 2004; Recommended for Publication by Dimitrios Tzovaras

\begin{abstract}
Manual pocket depth probing has been widely used as a retrospective diagnosis method in periodontics. However, numerous studies have questioned its ability to accurately measure the anatomic pocket depth. In this paper, an ultrasonic periodontal probing method is described, which involves using a hollow water-filled probe to focus a narrow beam of ultrasound energy into and out of the periodontal pocket, followed by automatic processing of pulse-echo signals to obtain the periodontal pocket depth. The signal processing algorithm consists of three steps: peak detection/characterization, peak classification, and peak identification. A dynamic wavelet fingerprint (DWFP) technique is first applied to detect suspected scatterers in the A-scan signal and generate a two-dimensional black and white pattern to characterize the local transient signal corresponding to each scatterer. These DWFP patterns are then classified by a two-dimensional FFT procedure and mapped to an inclination index curve. The location of the pocket bottom was identified as the third broad peak in the inclination index curve. The algorithm is tested on full-mouth probing data from two sequential visits of 14 patients. Its performance is evaluated by comparing ultrasonic probing results with that of full-mouth manual probing at the same sites, which is taken as the "gold standard."
\end{abstract}

Keywords and phrases: ultrasonic, periodontal, probe, wavelet.

\section{INTRODUCTION}

Most adults have a mild form of periodontal disease, while over 20 percent of older Americans have severe periodontal disease $[1,2,3]$. Periodontal disease involves the loss of tooth connective tissue (attachment) with subsequent destruction of tooth-supporting bone, leading to loss of teeth. In addition to being a major cause of tooth loss, periodontal disease has recently been associated with several systemic diseases. Animal and population-based studies have demonstrated an association between periodontal disease and diabetes, cardiovascular disease, stroke, and adverse pregnancy outcomes $[4,5,6]$. Despite the widespread problem of periodontal disease today, currently available diagnostic tests are limited in their effectiveness. None is a completely reliable indicator of periodontal disease activity and the best available diagnostic aid, probing pocket depths, is only a retrospective analysis of attachment already lost $[7,8,9,10,11,12,13]$. In traditional probing, which is now routinely done in the general dentistry office [14], a metal probe is inserted between the soft tissue of the gingival margin (gum line) and the tooth. Using fixed markings on the probe, the depth of probe penetration is typically measured relative to the gingival margin.

Numerous studies have questioned the ability of the periodontal probe to accurately measure the anatomic pocket depth $[15,16,17,18]$. The degree of probe tip penetration may be influenced by factors such as thickness of the probe, pressure applied, tooth contour, tooth position, presence of calculus, degree of periodontal inflammation, and the actual level of connective tissue fibers [19, 20, 21, 22, 23, 24]. As a result, probing measurements may overestimate attachment loss by as much as $2 \mathrm{~mm}$ in untreated sites, while underestimating attachment loss by an even greater margin following treatment $[25,26]$. The development of automated, controlled-force probes has reduced some of the operatorrelated error and subjectivity inherent in manual probing 
techniques $[27,28,29,30]$. However, standardized probing forces do not address anatomic and inflammatory factors $[31,32]$.

The first tests of ultrasonic imaging of the periodontal space attempted to image the crest of the alveolar bone by aiming the ultrasound transducer perpendicular to the long axis of the tooth $[33,34,35]$. While these efforts proved the feasibility of ultrasonic imaging in dentistry, this version of the technique could not detect periodontal attachment loss, and failed to gain clinical acceptance. Recently, researchers have begun exploring new uses of ultrasound in dentistry $[36,37,38,39]$ and studies have been conducted using ultrasound to image the periodontal pocket space by aiming the transducer apically into the pocket from the gingival margin $[40,41,42,43,44,45,46]$. The major technical barrier to this approach is providing an efficient coupling medium for the ultrasonic wave into the thin $(0.25-0.5 \mathrm{~mm})$ periodontal pocket. The probe described by Hinders and Companion uses a slight flow of water to couple the ultrasound wave into the pocket space. A hollow tip placed over the transducer narrows the ultrasonic beam, so that the beam is approximately the same width as the opening into the sulcus at the gingival margin.

The ultrasonic probe works by projecting a narrow, highfrequency $(10-15 \mathrm{MHz})$ ultrasonic pulse into the gingival sulcus/periodontal pocket, and then detecting echoes of the returning wave. The time series return signal (A-scan) can then be converted into a depth measurement by multiplying the time of arrival of the return signal by the speed of sound in water $(1500 \mathrm{~m} / \mathrm{s}$ ) and dividing by two (since the signal travels into the pocket and back). Ultrasonic probing is entirely painless, is as fast as manual probing, and has the potential to yield much more diagnostic information by providing the dentist and patient with a graphical representation of changes in pocket depth. However, due to the inherent complexity in the way ultrasonic waves interact with the inner structure of periodontal anatomy, it is unrealistic to train a dental hygienist to read out the pocket depth by watching and interpreting the echo waveform. Automated interpretation of these echoes is what enables a practical clinical system.

As an initial effort to automate interpretation of the echoes, a time-domain procedure was developed to simplify the waveforms and infer the depth of the periodontal pocket $[44,45,46]$. This procedure used a slope-detection algorithm to pick peaks in the A-scan signal, followed by smoothing and averaging operations to eliminate small random variations. The pocket depth was then inferred by dividing the simplified waveform into three regions and assuming the second transition from weaker peaks to noise is the estimation of the bottom of the periodontal pocket. Unfortunately, in many cases it was difficult or even impossible to define such three distinctive regions based on the echo amplitude. Even if the second transition point can be called out in some way, the depth of the periodontal pocket can only be inferred from it approximately. To overcome these limitations, information other than echo amplitude should be used to develop sensitive and reliable models to estimate the bottom of the periodontal pocket.

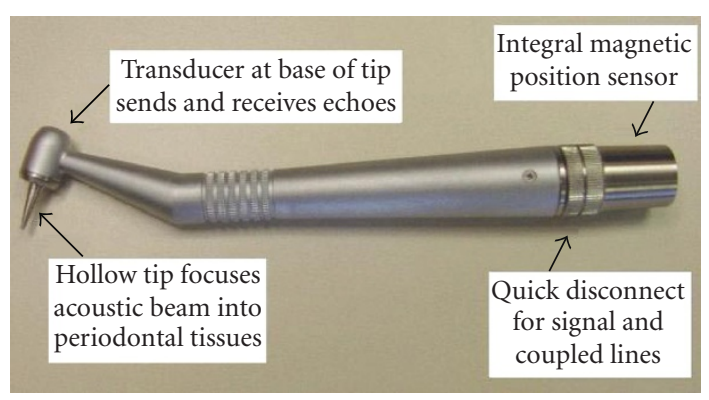

FIGURE 1: The ultrasonic probe handpiece.

The wavelet transform (WT) can be constructed as a bank of matched filters if the mother wavelet is chosen to be an efficient representation of the input signal itself, so it has been widely used to solve detection/estimation problems $[47,48,49,50,51]$. As a multi-resolution analysis approach, the WT is also an effective method to extract significant information from dynamic signals, which is often the key in automated signal classification applications $[52,53,54,55,56]$. To take advantage of this potential of the WT, a dynamic wavelet fingerprint technique [57] was adapted to develop a signal processing algorithm for the ultrasonic periodontal probe. In this approach, potential scatterers are first detected by picking peaks in the scale-averaged power (SAP) curve. A two-dimensional black and white pattern, called a DWFP, is then generated at each peak location to characterize the local transient signal. A two-dimensional FFT procedure is then applied to generate an inclination index for each DWFP pattern. The bottom of the periodontal pocket is then estimated from the third broad peak of the inclination index curve.

This paper is organized as follows. In Section 2, the experimental device and procedure are described. The algorithm used in this work is discussed in Section 3. The results are presented in Section 4. A discussion and conclusion is given in Section 5.

\section{CLINICAL INSTRUMENT AND SIGNALS}

The ultrasonic periodontal probe shown in Figure 1 is operated using a portable computer system. The probe itself includes a $10 \mathrm{MHz}$ piezocomposite transducer with a $2-\mathrm{mm}$ diameter active area. The transducer is housed within a contra-angled handpiece at the base of a hollow conical tip. The tip is designed to narrow the ultrasonic beam profile to $0.5 \mathrm{~mm}$ and to provide an area for water to sustain the ultrasonic wave and carry it into the periodontal pocket.

Figure 2 illustrates how the ultrasonic probe is used to explore the periodontal pocket. A stream of water is used as a couple to launch ultrasound energy into the periodontal pocket. The ultrasound wave interacts with the periodontal tissue and echoes carry relevant information back to the transducer. During operation, the ultrasonic probe is held in a pose similar to manual probing but with its tip touching the gingival margin. The ultrasonic probe is held in place momentarily at each of the standard probing locations to acquire a series of ultrasonic A-scan signals that are digitized 


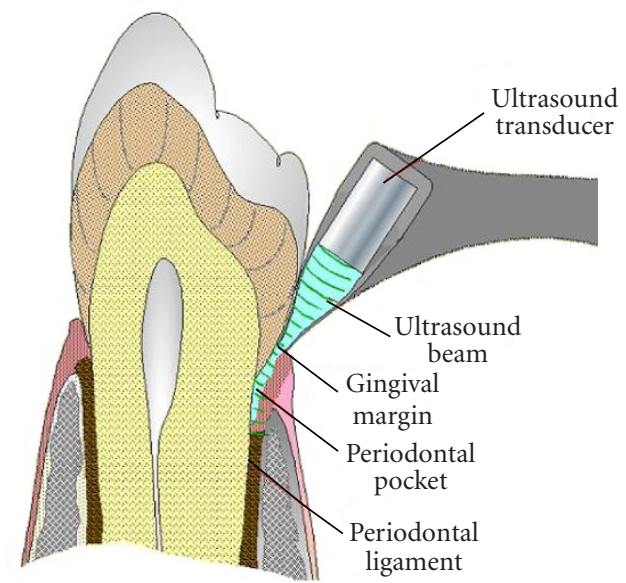

FIgURE 2: Schematic of ultrasonic periodontal probing.

and saved in the computer for later analysis. Water flow and data acquisition are automated via foot pedal control.

At Valley Periodontics, Appleton, Wisconsin, 14 patients were examined both by hand and by the ultrasonic instrument on two visits scheduled three months apart. For each patient, full-mouth probing was carried out, first by hand and then by the ultrasonic instrument. For each of up to 32 teeth for each patient, a periodontist performed probing at 6 sites (facial distal, facial middle, facial mesial, lingual distal, lingual middle, and lingual mesial), providing up to 192 corresponding ultrasonic and manual probing measurements per patient.

A typical A-scan signal obtained is shown in Figure 3a. The horizontal axis represents the time from 0 to $20.48 \mathrm{mi}-$ croseconds at $100 \mathrm{MHz}$ sampling rate. The vertical axis represents normalized voltage on an arbitrary scale digitized at 12 bits. The strong reflection region before point $2.5 \mathrm{mi}-$ croseconds arises from the echoes internal to the probe tip. After point 2.5 microseconds, it is the signal from the periodontal anatomy. We conclude this from a variety of phantom experiments as well as numerical simulations of the ultrasound propagation inside the tip that the first two are features of the tip and the third is from the anatomy. The work of Lynch [44] simulated and verified experimentally with phantoms and cadaver jaws what was due to the tip and what was due to the anatomy. Of course the results presented here will not definitively answer the question of some of what we are seeing is not the pocket base but instead calculus, tooth features, bone, and so forth. Future work is planned that should answer that and related questions definitively, via dog models as well as human testing where the gold standard is provided via ultrasonic scanning just prior to flap surgery or en bloc surgery followed by histological sectioning. Those studies will also help to understand other potential sources of probing error in addition to vertical position error.

\section{ALGORITHM DEVELOPMENT}

A flowchart of the proposed algorithm is shown in Figure 4, which involves three main steps: peak detection/

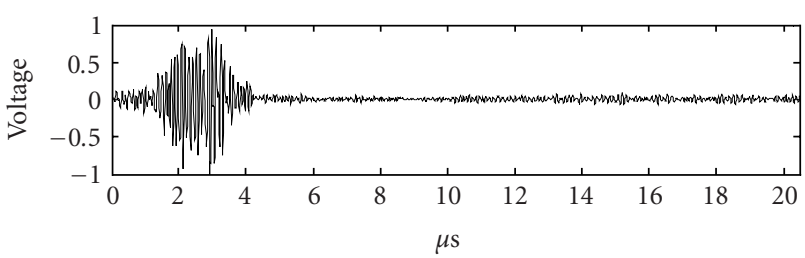

(a)

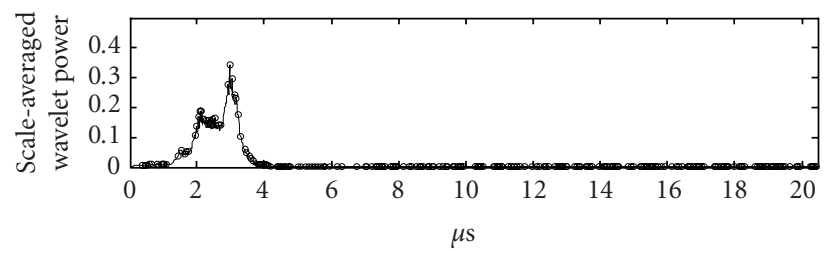

(b)

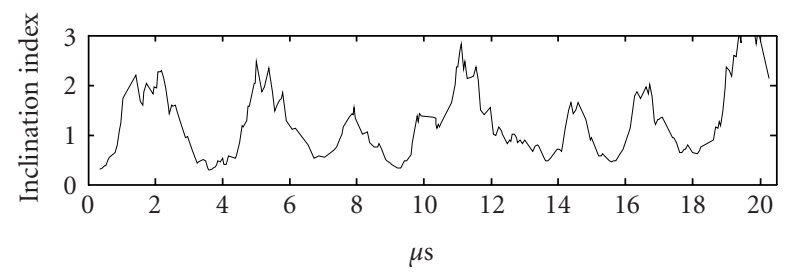

(c)

FIGURE 3: Illustration of signal processing for ultrasonic periodontal probing. (a) Original A-scan signal, (b) SAP peaks marked by the small circles, and (c) the inclination index curve. The third significant peak (at about 8 microseconds) corresponds to the estimated location of the bottom of the periodontal pocket. Vertical axes are arbitrary units.

characterization, peak classification, and peak identification.

A so-called dynamic wavelet fingerprint (DWFP) technique [57] was recently introduced by two of the authors. It consists of peak detection operation followed by peak characterization. The basic idea is to generate a simplified and intuitive two-dimensional pattern in time-scale domain for each detected transient signal, so that significant features can be extracted directly by watching the patterns or by using a modern pattern recognition technique. These features then can be used to identify specific signal of interest. The algorithm is explained as follows.

First, the scale-averaged wavelet power (SAP) proposed by Georgiou and Cohen [52] is calculated, that is,

$$
W_{a}^{2}(n)=\frac{1}{J} \sum_{j=1}^{J}\left|W\left(s_{j}, n\right)\right|^{2}
$$

where $W\left(s_{j}, n\right)$ are the continuous wavelet coefficients at scale $s_{j}$ and position $n$, that is,

$$
W\left(s_{j}, n\right)=\int_{R} f(t) \frac{1}{\sqrt{s_{j}}} \overline{\psi\left(\frac{t-n}{s_{j}}\right)} d t .
$$




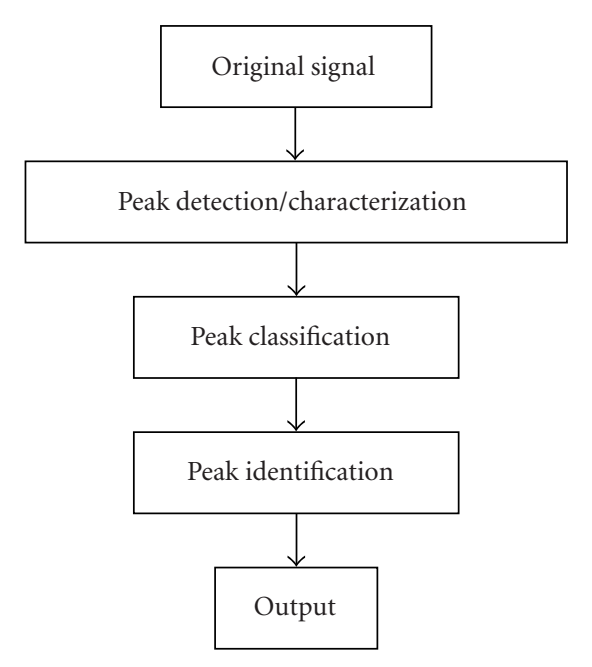

FIGURE 4: Flowchart of the algorithm used to process an ultrasound signal to detect the bottom of the periodontal pocket.

Here $f(t)$ is the echo waveform and $\psi$ is a mother wavelet. The results obtained by (1) are smoothed by a median filter to generate a SAP curve. SAP peaks are then picked out wherever the sign of the first derivative of the SAP curve changes from positive to negative as shown in Figure $3 \mathrm{~b}$. According to Georgiou and Cohen, the significant SAP peaks correspond to coherent scatterers that can be differentiated from the diffuse background. Assuming that the bottom of the periodontal pocket belongs to such coherent and resolvable scatterers, and that it can be detected as one of the SAP peaks, the question now is how to differentiate it from other scatterers.

To suppress noise and high-frequency interference that may cause distortion of the DWFP pattern generated later, a pruning procedure [58] based on the stationary wavelet transform [59] was applied on the original A-scan signal

$$
W_{S}(a, b)=0 \quad \text { for } a=1, \ldots, 5,
$$

where $W_{S}(a, b)$ is the stationary wavelet coefficients at scale $a$ and time $b$, that is, the wavelet coefficients at small scales are set to zero, which removes the highest frequencies. Next, a continuous wavelet transform is performed on the pruned A-scan signal using the Morlet wavelet $[60,61]$ :

$$
\psi(x)=C e^{-x^{2} / 2} \cos (5 x),
$$

where the constant $C$ is used for normalization. Different choices for the mother wavelet will, of course, give different DWFPs [57] with some better highlighting features of interest in the signals under study than others. For the ultrasonic periodontal probing data, the Morlet wavelet seems to give DWFP sequences dominated by "loop" features with varying inclination, which can be quantified in an automatic way.

For each peak in the SAP curve, the wavelet coefficients in its neighborhood are normalized into the range of $[-1,+1]$ and then projected onto the time-scale plane to generate

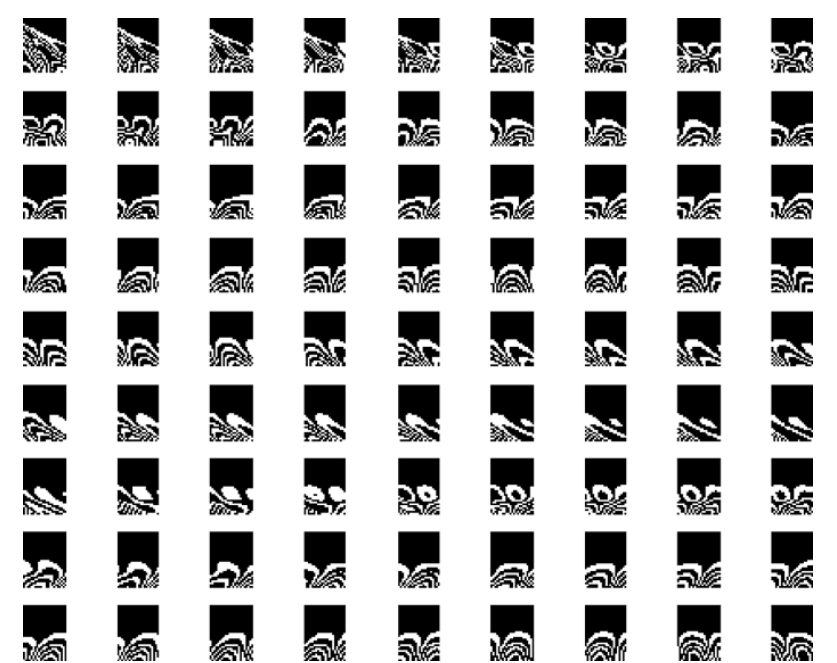

Figure 5: Typical DWFP sequence of an A-scan signal (time sequence: from left to right, from top to bottom).

a two-dimensional black and white pattern [57]. A typical DWFP sequence obtained is shown in Figure 5. In each frame of the sequence, time is the horizontal axis and wavelet scale is the vertical axis. This gives an abstract two-dimensional representation which allows "patterns" in the data to be recognized even when none are evident in the one-dimensional waveforms.

By observing many sets of DWFP sequences carefully, we noted that these DWFP patterns change their inclination regularly, that is, from left inclined to upright and to right inclined, and repeating. A two-dimensional FFT-based approach was designed to quantitatively characterize such variations.

Figure 6 shows three typical DWFP patterns from such a sequence along with their corresponding two-dimensional FFT images. For the right-inclined DWFP, it can be seen that its image is left diagonally dominated, that is, there are more bright pixels at the top-left and bottom-right corners than at the top-right and bottom-left corners. On the contrary, for the left-inclined DWFP, its image is right diagonally dominated. For the upright DWFP, its two-dimensional FFT image is almost symmetric.

Based on these observations, each two-dimensional FFT image is divided into two pairs of quadrants as shown in Figure $6 \mathrm{~d}$. An inclination index $I x$ is defined as the ratio of the number of white pixels in the shaded quadrants to that in the unshaded quadrants. The DWFP sequence is then mapped into an $I x$ curve as shown in Figure 3c. The regular variation of the DWFP is thus displayed as identifiable peaks and valleys.

To better explore the relationship between this intuitive pattern variation and the complex physics behind it, the same system was used to probe a simplified phantom built in a block of steel with holes of different depths (Figure 7). This sort of phantom is typically used to calibrate new automatic periodontal probes, and although it does not represent all 

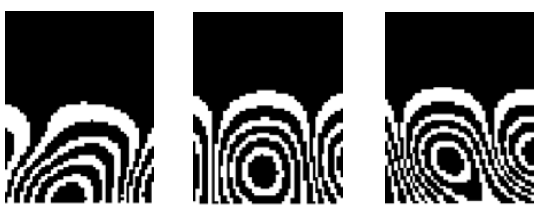

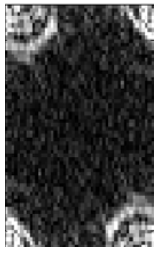

(a)

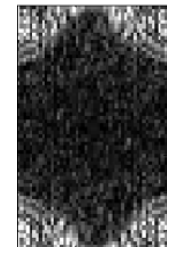

(b)

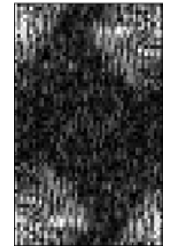

(c)

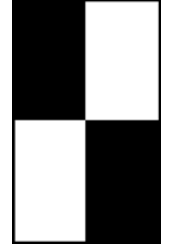

(d)
FIGURE 6: Two-dimensional FFT images of three typical DWFP patterns: (a) right-inclined DWFP, (b) upright DWFP, and (c) leftinclined DWFP. (d) Two regions used to calculate the inclination index of the two-dimensional FFT image.
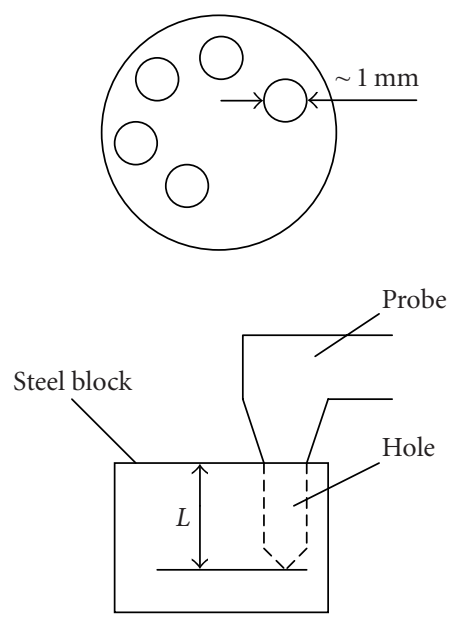

FIgure 7: Phantom used with the ultrasonic probe. The 1- mmdiameter holes range in depth from $L=2 \mathrm{~mm}$ to $L=9 \mathrm{~mm}$.

of the complicated periodontal pocket anatomy, it does provide us with an ultrasonically well-characterized system with which we can be certain that our algorithms are isolating true ultrasonic echoes from noise/artifacts inherent in the probing measurement.

Figure 8 illustrates an A-scan signal and its corresponding processing results. In the $I x$ curve, similar peaks and valleys can be seen as in Figure 3c. It is clear that the first significant peak corresponds to the reflection from the probe tip at about point 2.5 microseconds, and the third significant peak is close to the reflection from the bottom of the hole, which here is $5 \mathrm{~mm}$. As for the second significant peak in between, it is assumed to be caused by the interference of the water flow and the reflections of the wall of the hole. Accordingly, a qualitative explanation is proposed to describe the regular peaks and valleys in the $I x$ curve of the ultrasonic

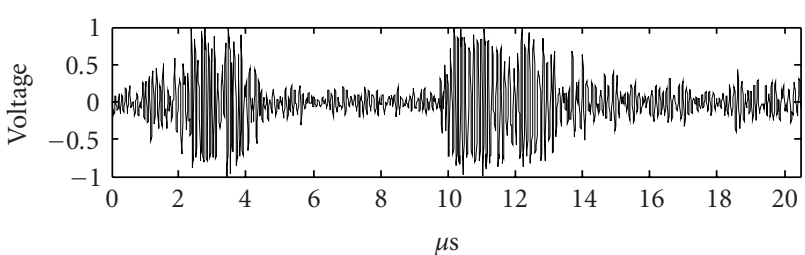

(a)

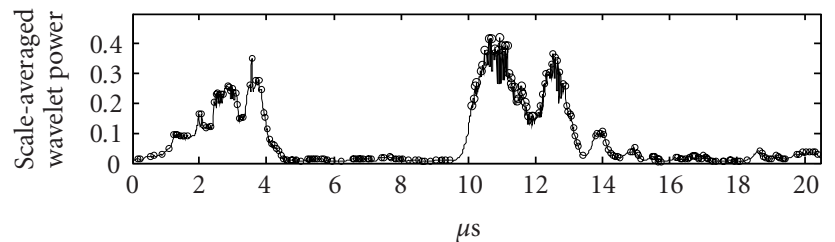

(b)

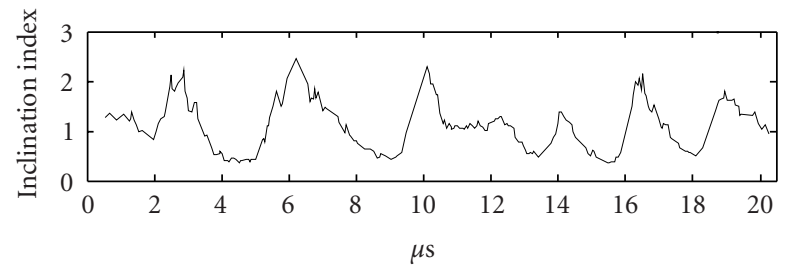

(c)

FIGURE 8: Phantom probing signal and corresponding processing results. (a) Original A-scan signal, (b) SAP peaks, (c) the inclination index curve. The third broad peak corresponds to the reflection from the bottom of the hole. Vertical axes are arbitrary units.

periodontal probing signal (shown in Figure 3c), the first significant peak arises from the probe tip, the second significant peak may be caused by the tooth surface, and the third significant peak may correspond to the bottom of the periodontal pocket. After the location of the bottom of the periodontal pocket is estimated as above, the pocket depth is calculated as the product of the time delay from the probe tip and the speed of ultrasound in water $(1.5 \mathrm{~mm} / \mu \mathrm{s})$, then divided by two.

\section{RESULTS}

A Matlab (The MathWorks, Inc.) program was developed to process full-mouth ultrasonic probing data of 14 patients acquired during two clinical sessions. It works in off-line mode and runs automatically until all of the digitized A-scan signals are processed.

Because of the lack of any ideal standard to compare with, we take the manual probing result as the "gold standard" and compare it with ultrasonic probing, keeping in mind that an accuracy of $\pm 1 \mathrm{~mm}$ for manual probing is perhaps being generous.

Firstly, ultrasonic probing readings are compared with manual probing readings at each probing site. If we allow, for the "error bar" of $\pm 0.5 \mathrm{~mm}, \pm 1.0 \mathrm{~mm}$, and $\pm 1.5 \mathrm{~mm}$, 


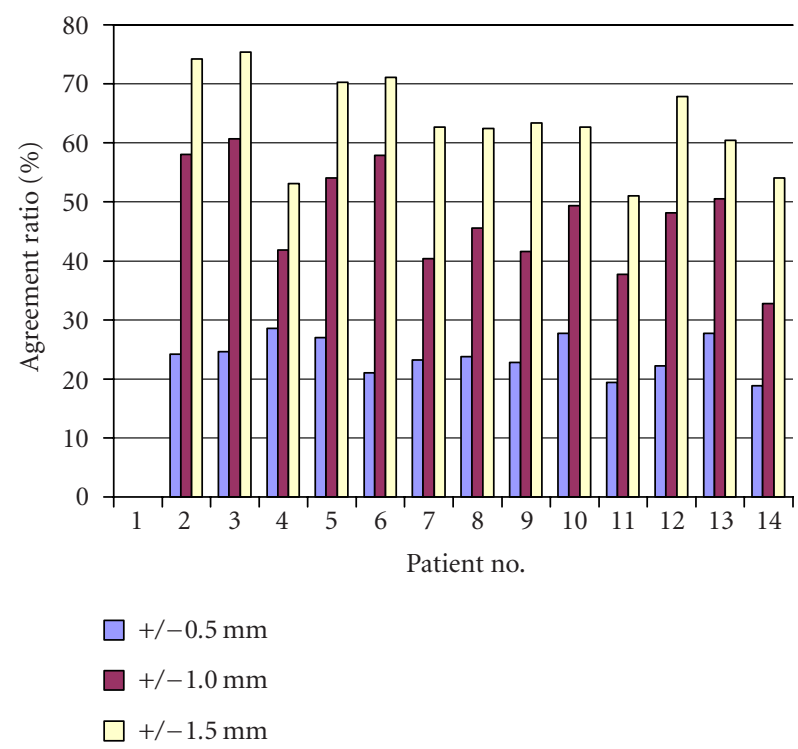

(a)

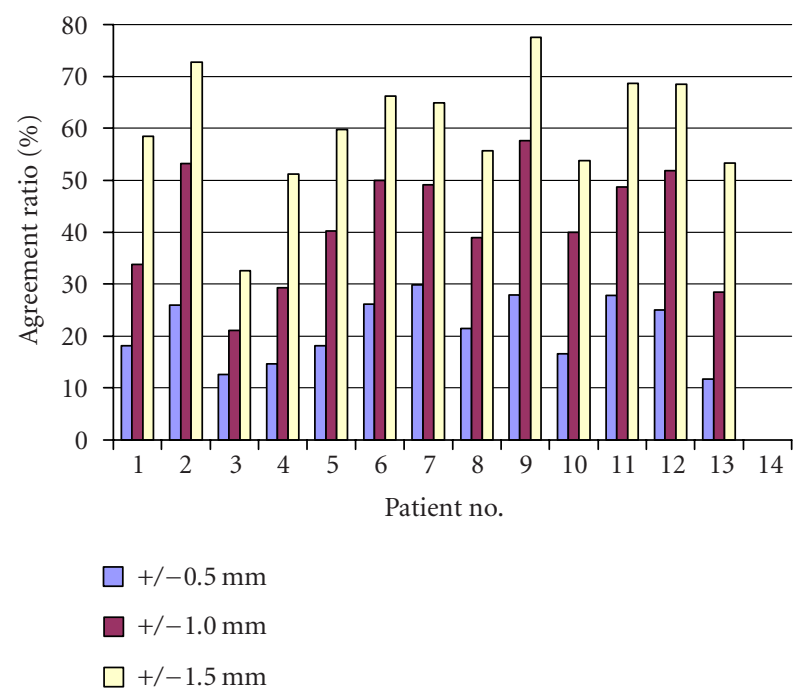

(b)

Figure 9: Ratio of agreement of ultrasonic probing versus manual probing. (a) May 18, 2001, and (b) August 17, 2001. Note that the results for patient 1 in (a) and patient 14 in (b) are unavailable due to personal absence in corresponding clinical visit.

respectively, the agreement ratio (number of ultrasonic probing measurements within the "error bar" divided by the number of total ultrasonic probing results) is about $20 \%$, $40 \%$, and $60 \%$, correspondingly, as shown in Figure 9.

Statistically, the agreement between ultrasonic probing and manual probing was evaluated by the Bland-Altman method [62].

As an example, a plot of the difference between the methods and their means was drawn for patient 2, May 18, in Figure 10. It seems that the difference does not increase with the mean. The regular gaps are due to integer measurements

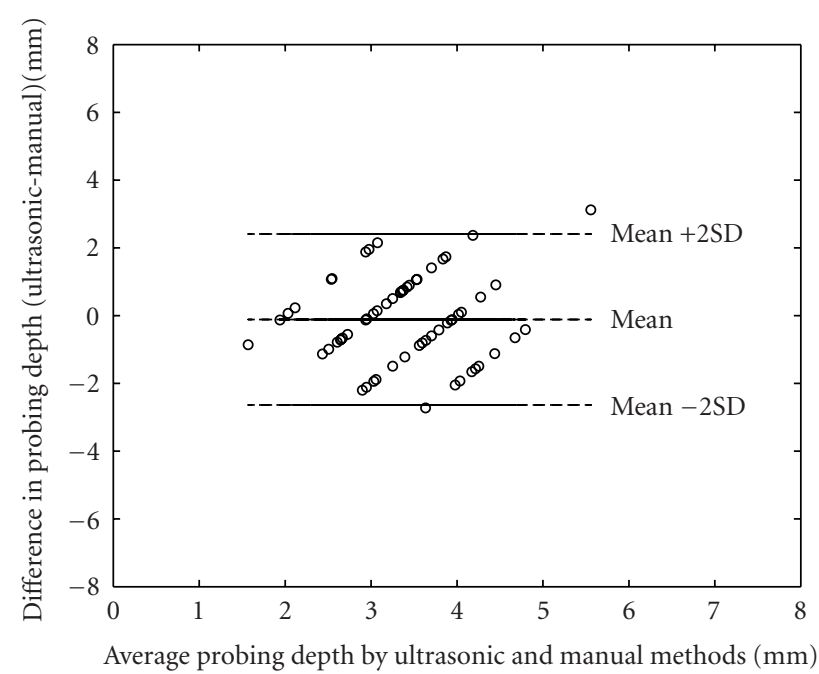

FIGURE 10: Difference against mean for periodontal probing depth of patient 2, May 18 .

of manual probing. Assuming the difference is normally distributed, the mean difference $\bar{d}$ and the standard deviation SD of the difference are calculated as $\bar{d}=-0.1128$ and $\mathrm{SD}=1.2611$. The "limits of agreement" can be obtained as

$$
\begin{aligned}
& \bar{d}-2 \mathrm{SD}=-0.1128-(2 \times 1.2611)=-2.635 \mathrm{~mm}, \\
& \bar{d}+2 \mathrm{SD}=-0.1128+(2 \times 1.2611)=2.409 \mathrm{~mm} .
\end{aligned}
$$

According to Bland and Altman, about 95\% of differences will lie between these limits. In other words, the ultrasonic probing depth may be $2.6 \mathrm{~mm}$ below or $2.4 \mathrm{~mm}$ above the manual probing depth. The precision of the estimation of $\bar{d}, \bar{d}+2$ SD, and $\bar{d}-2$ SD can be evaluated by using $95 \%$ confidence interval of a $t$-distribution with $n-1$ degree of freedom, where $n$ is the sample size. For the patient chosen above, these confidence intervals were obtained as $[-0.3818,0.1563],[1.9433,2.8754]$, and [ $-3.1010,-2.1689]$, respectively. Similar calculations were performed on other data sets and the results were presented in Tables 1 and 2 .

Taking the nonzero $\bar{d}$ into consideration, the limits of agreement are generally in the range of $\pm 3 \mathrm{~mm}$. This may indicate a lack of agreement between ultrasonic probing and manual probing, which could be caused by several factors. The specific anatomical features measured by the two methods may be different; the probing site and angle may not be exactly the same, and so forth $[63,64,65,66]$. Of course, it may arise also from the model used in the algorithm. As mentioned above, the regular variation of the inclination of the DWFP patterns is motivated by simulations, cadaver jaw studies, and an experiment on a steel block phantom, which is not entirely representative of the problem at hand, even though it does eliminate the sizeable error in the manual probing gold standard. To better understand the regular variation of the DWFP patterns, a more accurate model should be developed guided by systematic clinical experiments carried out in the future. 
TABle 1: (a) Mean difference $\bar{d}$ and its 95\% confidence intervals (May 18). (b) Limits of agreement $\bar{d} \pm 2$ SD and their 95\% confidence intervals (May 18). N/A: patient not available.

(a)

\begin{tabular}{|c|c|c|c|c|c|c|c|c|c|c|c|c|c|c|}
\hline Patient no. & - 1 & 2 & 3 & 4 & 5 & 6 & 7 & 8 & 9 & 10 & 11 & 12 & 13 & 14 \\
\hline $\bar{d}$ & $1 \mathrm{~V} / \mathrm{A}$ & -0.1128 & 0.4532 & 0.1722 & 0.8217 & 0.0176 & -0.7202 & 0.2462 & 0.1853 & -0.7140 & -0.3904 & -0.0353 & -0.6021 & -1.2224 \\
\hline Low limit & N/A & -0.3818 & 0.1741 & -0.1305 & 0.5303 & -0.3553 & -1.0045 & -0.0501 & -0.0714 & -0.9960 & -0.7110 & -0.3243 & -0.8485 & -1.4429 \\
\hline High limit & N/A & 0.1563 & 0.7323 & 0.4750 & 1.1130 & 0.3905 & -0.4360 & 0.5425 & 0.4421 & -0.4320 & -0.0697 & 0.2538 & -0.3557 & -1.0019 \\
\hline
\end{tabular}

(b)

\begin{tabular}{|c|c|c|c|c|c|c|c|c|c|c|c|c|c|c|}
\hline Patient no. & . 1 & 2 & 3 & 4 & 5 & 6 & 7 & 8 & 9 & 10 & 11 & 12 & 13 & 14 \\
\hline$+2 \mathrm{SD}$ & $\mathrm{J} / \mathrm{A}$ & 4094 & 3.0481 & 7399 & 3.8056 & 1 & 2 & 4 & 4 & 7 & 36 & 3.0 & 3459 & 1.6769 \\
\hline Low limit & N/A & 1.9433 & 2.5647 & 3.2156 & 3.3009 & 2.1082 & 2.1542 & 3.2782 & 2.8127 & 1.8562 & 2.8332 & 2.5612 & 1.9191 & 1.2950 \\
\hline High & N/A & 2.8754 & 3.5315 & 13 & 4.3103 & 3.3 & 3 & 47 & 3.7 & 2.8331 & 3.9 & 3.5 & 2.7727 & 2.0588 \\
\hline$\overline{\bar{d}}-2 \mathrm{SD}$ & N/A & 349 & -2.1417 & -3.3955 & -2.1623 & -2.7188 & -4.0869 & -3.2991 & -2.8867 & -3.7726 & -4.1693 & -3.1324 & -3.5502 & -4.1217 \\
\hline Low limit & N/A & 10 & -2.6 & -3 & -2 & -3 & - & 23 & - & 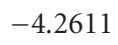 & -4 & 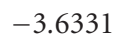 & 7 & -4.5036 \\
\hline High limit & N/A & -2.1689 & -1.6583 & -2.8712 & -1.6576 & -2.0729 & -3.5 & -2.7858 & -2.4420 & -3.2842 & -3 . & -2 & 234 & -3.7398 \\
\hline
\end{tabular}

TABle 2: (a) Mean difference $\bar{d}$ and its 95\% confidence intervals (August 17). (b) Limits of agreement $\bar{d} \pm 2$ SD and their 95\% confidence intervals (August 17). N/A: patient not available.

(a)

\begin{tabular}{|c|c|c|c|c|c|c|c|c|c|c|c|c|c|c|}
\hline Patient no. & 1 & 2 & 3 & 4 & 5 & 6 & 7 & 8 & 9 & 10 & 11 & 12 & 13 & 14 \\
\hline $\bar{d}$ & -0.0383 & 0.4431 & 2.1375 & 0.0154 & -0.7111 & 0.4561 & 0.1884 & 0.9767 & 0.5739 & 1.2619 & -0.0827 & 0.1696 & 1.3116 & N/A \\
\hline Low limit & -0.3708 & 0.2035 & 1.9106 & -0.5105 & -1.0207 & 0.2277 & -0.0732 & 0.7546 & 0.3573 & 1.0374 & -0.3309 & -0.0641 & 1.0875 & N/A \\
\hline High limit & 0.2941 & 0.6826 & 2.3644 & 0.5414 & -0.4015 & 0.6844 & 0.4500 & 1.1989 & 0.7905 & 1.4863 & 0.1654 & 0.4034 & 1.5356 & N/A \\
\hline
\end{tabular}

(b)

\begin{tabular}{lcccccccccccccc}
\hline Patient no. & 1 & 2 & 3 & 4 & 5 & 6 & 7 & 8 & 9 & 10 & 11 & 12 & 13 & 14 \\
\hline $\bar{d}+2$ SD & 3.4348 & 2.9452 & 4.7704 & 4.0246 & 2.5230 & 3.5555 & 3.5133 & 4.2047 & 3.2903 & 4.4792 & 3.0848 & 3.0615 & 4.4338 & N/A \\
Low limit & 2.8589 & 2.5303 & 4.3774 & 3.1137 & 1.9868 & 3.1600 & 3.0602 & 3.8200 & 2.9152 & 4.0904 & 2.6551 & 2.6567 & 4.0457 & N/A \\
High limit & 4.0106 & 3.3601 & 5.1634 & 4.9356 & 3.0592 & 3.9510 & 3.9664 & 4.5895 & 3.6655 & 4.8679 & 3.5146 & 3.4664 & 4.8219 & N/A \\
\hline$\overline{\bar{d}}-2$ SD & -3.5115 & -2.0591 & -0.4953 & -3.9938 & -3.9452 & -2.6434 & -3.1365 & -2.2513 & -2.1425 & -1.9554 & -3.2503 & -2.7223 & -1.8107 & N/A \\
Low limit & -4.0873 & -2.4739 & -0.8884 & -4.9048 & -4.4814 & -3.0389 & -3.5895 & -2.6360 & -2.5176 & -2.3441 & -3.6801 & -3.1271 & -2.1988 & N/A \\
High limit & -2.9356 & -1.6442 & -0.1023 & -3.0828 & -3.4089 & -2.2479 & -2.6834 & -1.8665 & -1.7674 & -1.5667 & -2.8206 & -2.3174 & -1.4226 & N/A
\end{tabular}

\section{CONCLUSION}

An ultrasonic periodontal probing instrument is being developed. It uses a hollow water-filled tip to couple the ultrasound energy into and back out of the periodontal pocket, thus probing the periodontal anatomy by a non-invasive, painless, and automatic technique. A key to automation of the probing is an ultrasonic signal processing algorithm for the periodontal probing instrument, which uses a dynamic wavelet fingerprint technique to detect and characterize the transient signals that arise from each suspected scatterer and estimate the location of the echoes corresponding to the bottom of the periodontal pocket.
Clinical data from 14 patients have been processed with the proposed algorithm. Site-by-site comparison shows about $40 \%$ agreement ratio between ultrasonic and manual probing at the tolerance of $\pm 1.0 \mathrm{~mm}$. Statistically, however, lack of agreement between ultrasonic and manual probing was found in terms of the limits of agreement proposed by Bland and Altman. It may arise from the model used in the algorithm and further research is necessary to develop more accurate phantom and understand the physics behind the intuitive variation of the DWFP patterns. In other work using variations of this DWFP technique $[67,68,69]$ we have found that choosing the best mother wavelet allows interesting patterns to be highlighted in the resulting 
DWFP which are then amenable to automated interpretation.

We have planned for the future additional studies which will enroll much larger numbers of patients. We note that with 32 teeth per patient and six probing sites per tooth 14 patients tested twice gives around 5000 individual direct comparisons between manual and ultrasonic probing. The site-specific nature of periodontal disease makes those independent comparisons. For example, a 500-patient study would give about 50000 such comparisons. Future studies are also planned that are perhaps closer to an ideal test, via dog models as well as human testing where the gold standard is provided via ultrasonic scanning just prior to flap surgery or en bloc surgery. Those studies will also help to understand other potential sources of probing error in addition to the vertical position error considered here. We have also planned tests which would ultrasonically probe condemned teeth prior to extraction. It will be important to also continue to compare with standard manual (and controlledforce) probing so that we understand how this new measurement correlates with what is standard practice clinically now.

\section{ACKNOWLEDGMENTS}

The authors would like to thank Jonathan Stevens for constructing much of the instrumentation and the new handpiece. This work was supported by the National Institute of Dental and Craniofacial Research (no. 1R15DE12851-01 and no. 1R43DE1255701-A2) and by Virginia's Center for Innovative Technology (no. BIO-01-004).

\section{REFERENCES}

[1] C. A. Evans, D. V. Kleinman, W. R. Maas, et al., "Oral health in America: a report of the Surgeon General," Tech. Rep. National Institutes of Health (NIH) publication no. 00-4713, National Institute of Dental and Craniofacial Research, Bethesda, Md, USA, 2000.

[2] E. P. Barrington and M. Nevins, "Diagnosing periodontal diseases," J. Am. Dent. Assoc., vol. 121, no. 4, pp. 460-464, 1990.

[3] G. C. Armitage, "Diagnosis of periodontal diseases," J. Periodontol., vol. 74, no. 8, pp. 1237-1247, 2003.

[4] E. B. Fowler, L. G. Breault, and M. F. Cuenin, "Periodontal disease and its association with systemic disease," Mil. Med., vol. 166, no. 1, pp. 85-89, 2001.

[5] D. W. Paquette, "The periodontal infection-systemic disease link: a review of the truth or myth," J. Int. Acad. Periodontol., vol. 4, no. 3, pp. 101-109, 2002.

[6] J. Slots, "Update on general health risk of periodontal disease," Int. Dent. J., vol. 53, no. (Suppl 3), pp. 200-207, 2003.

[7] A. D. Haffajee, S. S. Socransky, and J. M. Goodson, "Clinical parameters as predictors of destructive periodontal disease activity," J. Clin. Periodontol., vol. 10, no. 3, pp. 257-265, 1983.

[8] J. F. Tessier, G. V. Kulkarni, R. P. Ellen, and C. A. McCulloch, "Probing velocity: novel approach for assessment of inflamed periodontal attachment," J. Periodontol., vol. 65, no. 2, pp. 103-108, 1994.
[9] P. S. Hull, V. Clerehugh, and A. Ghassemi-Aval, "An assessment of the validity of a constant force electronic probe in measuring probing depths," J. Periodontol., vol. 66, no. 10, pp. 848-851, 1995.

[10] T. E. Rams and J. Slots, "Comparison of two pressure-sensitive periodontal probes and a manual periodontal probe in shallow and deep pockets," Int. J. Periodontics Restorative Dent., vol. 13, no. 6, pp. 520-529, 1993.

[11] N. P. Lang and E. F. Corbet, "Periodontal diagnosis in daily practice," Int. Dent. J., vol. 45, no. 1, pp. 3-15, 1995.

[12] G. Greenstein and I. Lamster, "Understanding diagnostic testing for periodontal diseases," J. Periodontol., vol. 66, no. 8, pp. 659-666, 1995.

[13] M. A. Listgarten, "Periodontal probing: what does it mean?," J. Clin. Periodontol., vol. 7, no. 3, pp. 165-176, 1980.

[14] J. P. Trovato, "The role of the general dentist in periodontal care," Gen. Dent., vol. 51, no. 2, pp. 176-181, 2003.

[15] L. Tupta-Veselicky, P. Famili, F. J. Ceravolo, and T. Zullo, "A clinical study of an electronic constant force periodontal probe," J. Periodontol., vol. 65, no. 6, pp. 616-622, 1994.

[16] M. E. Palou, M. J. McQuade, and J. A. Rossmann, "The use of ultrasound for the determination of periodontal bone morphology," J. Periodontol., vol. 58, no. 4, pp. 262-265, 1987.

[17] F. Hunter, "Periodontal probes and probing," Int. Dent. J., vol. 44, no. (5 Suppl 1), pp. 577-583, 1994.

[18] L. Mayfield, G. Bratthall, and R. Attstrom, "Periodontal probe precision using 4 different periodontal probes," J. Clin. Periodontol., vol. 23, no. 2, pp. 76-82, 1996.

[19] M. K. Jeffcoat, "Machines in periodontics," Alpha Omegan, vol. 84, no. 4, pp. 18-20, 22, 1991.

[20] M. Quirynen, A. Callens, D. van Steenberghe, and M. Nys, "Clinical evaluation of a constant force electronic probe," $J$. Periodontol., vol. 64, no. 1, pp. 35-39, 1993.

[21] M. Cattabriga, "Future diagnostic possibilities in periodontology," Int. Dent. J., vol. 43, no. 2, pp. 109-115, 1993.

[22] S. G. Grossi, R. G. Dunford, A. Ho, G. Koch, E. E. Machtei, and R. J. Genco, "Sources of error for periodontal probing measurements," J. Periodontal Res., vol. 31, no. 5, pp. 330336, 1996

[23] D. S. Barendregt, U. Van der Velden, J. Reiker, and B. G. Loos, "Clinical evaluation of tine shape of 3 periodontal probes using 2 probing forces," J. Clin. Periodontol., vol. 23, no. 4, pp. 397-402, 1996.

[24] J. J. Garnick and L. Silverstein, "Periodontal probing: probe tip diameter," J. Periodontol., vol. 71, no. 1, pp. 96-103, 2000.

[25] S. F. Wang, K. N. Leknes, G. J. Zimmerman, T. J. Sigurdsson, U. M. Wikesjo, and K. A. Selvig, "Reproducibility of periodontal probing using a conventional manual and an automated force-controlled electronic probe," J. Periodontol., vol. 66, no. 1, pp. 38-46, 1995.

[26] J. F. Tessier, R. P. Ellen, P. Birek, G. V. Kulkarni, and C. A. McCulloch, "Relationship between periodontal probing velocity and gingival inflammation in human subjects," J. Clin. Periodontol., vol. 20, no. 1, pp. 41-48, 1993.

[27] L. Tupta-Veselicky, P. Famili, F. J. Ceravolo, and T. Zullo, "A clinical study of an electronic constant force periodontal probe," J. Periodontol., vol. 65, no. 6, pp. 616-622, 1994.

[28] M. Cattabriga, "Future diagnostic possibilities in periodontology," Int. Dent. J., vol. 43, no. 2, pp. 109-115, 1993.

[29] M. C. K. Yang, R. G. Marks, I. Magnusson, B. Clouser, and W. B. Clark, "Reproducibility of an electronic probe in relative attachment level measurements," J. Clin. Periodontol., vol. 19, no. 8, pp. 541-548, 1992. 
[30] N. Ahmed, T. L. P. Watts, and R. F. Wilson, "An investigation of the validity of attachment level measurements with an automated periodontal probe," J. Clin. Periodontol., vol. 23, no. 5, pp. 452-455, 1996.

[31] A. Aguero, J. J. Garnick, J. Keagle, D. E. Steflik, and W. O. Thompson, "Histological location of a standardized periodontal probe in man," J. Periodontol., vol. 66, no. 3, pp. 184 190, 1995.

[32] J. G. Keagle, J. J. Garnick, J. R. Searle, and W. O. Thompson, "Effect of gingival wall on resistance to probing forces," J. Clin. Periodontol., vol. 22, no. 12, pp. 953-957, 1995.

[33] H. Fukukita, T. Yano, A. Fukumoto, K. Sawada, T. Fujimasa, and I. Sunada, "Development and application of an ultrasonic imaging system for dental diagnosis," J. Clin. Ultrasound, vol. 13, no. 8, pp. 597-600, 1985.

[34] C. Lost, K. M. Irion, and W. Nussle, "Periodontal ultrasonic diagnosis: experiments on thin bony platelets and on a simulated periodontal ligament space," J. Periodontal Res., vol. 23, no. 6, pp. 347-351, 1988.

[35] M. E. Palou, M. J. McQuade, and J. A. Rossmann, "The use of ultrasound for the determination of periodontal bone morphology," J. Periodontol., vol. 58, no. 4, pp. 262-265, 1987.

[36] S. Y. Ng, A. Songra, N. Ali, and J. L. B. Carter, "Ultrasound features of osteosarcoma of the mandible-a first report," Oral Surg. Oral Med. Oral Pathol. Oral Radiol. Endod., vol. 92, no. 5, pp. 582-586, 2001.

[37] N. Hamano, K. Hanaoka, K. Ebihara, M. Toyada, and T. Teranaka, "Evaluation of adhesive defects using an ultrasonic pulse-reflection technique," Dent. Mater., vol. 22, no. 1, pp. 66-79, 2003

[38] S. R. Ghorayeb and T. Valle, "Experimental evaluation of human teeth using noninvasive ultrasound: echodentography," IEEE Trans. Ultrason., Ferroelect., Freq. Contr., vol. 49, no. 10, pp. 1437-1443, 2002.

[39] F. I. Tsiolis, I. G. Needleman, and G. S. Griffiths, "Periodontal ultrasonography," J. Clin. Periodontol., vol. 30, no. 10, pp. 849-854, 2003

[40] A. C. Lassal and P. A. Payne, "Scanning and imaging using Lamb waves," Acoustical Imaging, vol. 23, pp. 355-361, 1997.

[41] J. A. Companion, "Differential measurement periodontal structures mapping system," US Patent \#5,755,571, 1998.

[42] D. R. Loker and K. Hagenbuch, "Ultrasonic periodontal diagnostic instrumentation system with clinical results," Journal of the International Measurement Confederation, vol. 23, no. 3, pp. 125-129, 1998.

[43] M. K. Hinders and J. Companion, "Ultrasonic periodontal probe," in Review of Progress in Quantitative Nondestructive Evaluation (QNDE'99), D. O. Thompson and D. E. Chimenti, Eds., vol. 18, pp. 1609-1615, Kluwer Academic/Plenum Publishers Snowbird, Utah, July 1998.

[44] J. E. Lynch, Development and clinical testing of an ultrasonographic periodontal probe, Ph.D. dissertation, College of William \& Mary, Williamsburg, Va, USA, 2001.

[45] M. K. Hinders, J. E. Lynch, and G. McCombs, "Clinical tests of an ultrasonic periodontal probe," in Review of Progress in Quantitative Nondestructive Evaluation (QNDE '01), D. O. Thompson and D. E. Chimenti, Eds., vol. 21, pp. 1880-1887, American Institute of Physics Brunswick, ME, USA, JulyAugust 2001.

[46] J. E. Lynch and M. K. Hinders, "Ultrasonic device for measuring periodontal attachment levels," Review of Scientific Instruments, vol. 73, no. 7, pp. 2686-2693, 2002.

[47] J. Rosiene and H. Sholl, "Application of wavelets to ultrasonic evaluation of thickness," in Wavelet Applications, vol. 2242 of SPIE Proceedings, pp. 488-498, Orlando, Fla, USA, April 1994.
[48] A. Abbate, J. Koay, J. Frankel, S. C. Schroeder, and P. Das, "Application of wavelet transform signal processor to ultrasound," in Proc. IEEE Ultrasonic Symposium, vol. 2, pp. 11471152, Cannes, France, November 1994.

[49] R. N. Strickland and H. Hahn, "Wavelet transforms for detecting microcalcifications in mammograms," IEEE Trans. Med. Imag., vol. 15, no. 2, pp. 218-229, 1996.

[50] X. L. Xu, A. H. Tewfik, and J. F. Greenleaf, "Time delay estimation using wavelet transform for pulsed-wave ultrasound," Ann. Biomed. Eng., vol. 23, no. 5, pp. 612-621, 1995.

[51] X. L. Xu and J. F. Greenleaf, "Wavelet transform for cross correlation processing in PW ultrasound," in Proc. 16th Annual International Conference of the IEEE Engineering in Medicine and Biology Society. Engineering Advances: New Opportunities for Biomedical Engineers, vol. 2, pp. 1224-1225, Baltimore, Md, USA, November 1994.

[52] G. Georgiou and F. S. Cohen, "Tissue characterization using the continuous wavelet transform I. Decomposition method," IEEE Trans. Ultrason., Ferroelect., Freq. Contr., vol. 48, no. 2, pp. 355-363, 2001.

[53] M. Szczuka and P. Wojdyllo, "Neuro-wavelet classifiers for EEG signals based on rough set methods," Neurocomputing, vol. 36, no. 1-4, pp. 103-122, 2001.

[54] Z. Dokur and T. Olmez, "ECG beat classification by a novel hybrid neural network," Comput. Methods Programs Biomed., vol. 66, no. 2-3, pp. 167-181, 2001.

[55] S. Legendre, D. Massicotte, J. Goyette, and T. K. Bose, "Neural classification of Lamb wave ultrasonic weld testing signals using wavelet coefficients," IEEE Trans. Instrum. Meas., vol. 50, no. 3, pp. 672-678, 2001.

[56] M. Lemistre and D. Balageas, "Structural health monitoring system based on diffracted Lamb wave analysis by multiresolution processing," Smart Materials and Structures, vol. 10, no. 3, pp. 504-511, 2001.

[57] J. D. Hou and M. K. Hinders, "Dynamic wavelet fingerprint identification of ultrasound signals," Materials Evaluation, vol. 60, pp. 1089-1093, 2002.

[58] A. Abbate, J. Koay, J. Frankel, S. C. Schroeder, and P. Das, "Signal detection and noise suppression using a wavelet transform signal processor: application to ultrasonic flaw detection," IEEE Trans. Ultrason., Ferroelect., Freq. Contr., vol. 44, no. 1, pp. 14-26, 1997.

[59] R. R. Coifman and D. L. Donoho, "Translation invariant denoising," in Wavelets and Statistics, vol. 103 of Lecture Notes in Statistics, pp. 125-150, Springer-Verlag, New York, NY, USA, 1995.

[60] I. Daubechies, Ten Lectures on Wavelets, SIAM, Philadelphia, $\mathrm{Pa}, \mathrm{USA}, 1992$.

[61] User's Guide, MATLAB Wavelet Toolbox, The MathWorks, 2000.

[62] J. M. Bland and D. G. Altman, "Statistical methods for assessing agreement between two methods of clinical measurement," Lancet, vol. 1, no. 8476, pp. 307-310, 1986.

[63] T. L. P. Watts, "Constant force probing with and without a stent in untreated periodontal disease: the clinical reproducibility problem and possible sources of error," J. Clin. Periodontol., vol. 14, no. 7, pp. 407-411, 1987.

[64] T. L. P. Watts, "Probing site configuration in patients with untreated periodontitis. A study of horizontal positional error," J. Clin. Periodontol., vol. 16, no. 8, pp. 529-533, 1989.

[65] T. L. P. Watts, C. F. Beards, P. D. Ewing, and S. Leeman, "Periodontal disease activity: a development strategy for its investigation by means of accurate 3-dimensional clinical measurement," J. Clin. Periodontol., vol. 22, no. 3, pp. 201-207, 1995. 
[66] J. C. Edge, T. L. P. Watts, and P. D. Ewing, "Initial fourth generation periodontal probing in human beings," J. Dent. Res., vol. 78, pp. 1045, 1999.

[67] J. D. Hou, Ultrasonic signal detection and characterization using dynamic wavelet fingerprints, Ph.D. dissertation, Department of Applied Science, College of William \& Mary, Williamsburg, Va, USA, April 2004.

[68] J. D. Hou, K. R. Leonard, and M. K. Hinders, "Automatic multi-mode Lamb wave arrival time extraction for improved tomographic reconstruction," Inverse Problems, vol. 20, no. 6, pp. 1873-1888, 2004.

[69] M. K. Hinders, J. D. Hou, and J. C. P. McKeon, "Ultrasonic inspection of thin multilayers," to appear in Review of Progress in Quantitative Nondestructive Evaluation, D. O. Thompson and D. E. Chimenti, Eds., vol. 24, 2005.

Jidong Hou received a B.S. degree in mechanical engineering from Institute of Armoured Vehicle Engineering, Beijing, China in 1991 and an M.S. degree in materials engineering from University of National Defense Science and Technology, Changsha, China in 1994. He received his M.S. degree in 2002 and Ph.D. degree in 2004, both in nondestructive evaluation from College of William \& Mary, Virginia. Since 2002, he

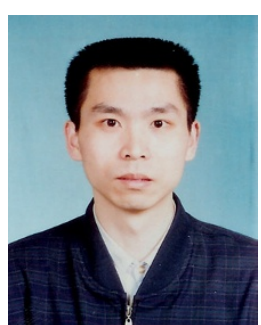
has been working at Sonix, Inc., Springfield, Va. His research interests include ultrasonic signal processing and imaging, and ultrasonic instrumentation.

S. Timothy Rose is a periodontist in private practice in Appleton, Wisconsin. He received his B.A. degree from Ohio State University, a D.D.S. degree from Ohio State University College of Dentistry, M.S. degree from Ohio State University, and a professional postgraduate certificate from the Department of Periodontology, College of Dentistry, Ohio State University. He is the past President of the American Academy of Periodontology and the American Dental Association. He is also past President of the Wisconsin Dental Association, the Wisconsin Society of Periodontists, and the Midwest Society of Periodontology. He served on the Governor's task force on the future of dentistry in Wisconsin. He has also been Chairman of the ADA's committee on information technology and has served as USA National Secretary for the World Dental Federation (FDI).

Mark K. Hinders holds B.S., M.S., and Ph.D. degrees in aerospace and mechanical engineering from Boston University, and is currently an Associate Professor of applied science at the College of William and Mary in Virginia. Before coming to Williamsburg in 1993, Professor Hinders was a Senior Scientist at Massachusetts Technological Laboratory, Inc., and also a Research Assistant Professor at Boston University. Before that

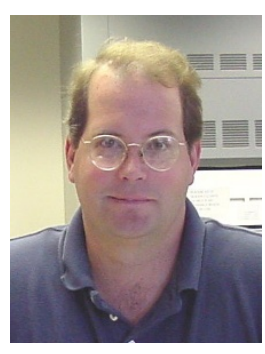
he was an Electromagnetics Research Engineer at the USAF Rome Laboratory located at Hanscom AFB, Mass. Professor Hinders conducts research in wave propagation and scattering phenomena, applied to robotics, medical imaging, remote sensing, and nondestructive evaluation. In particular, he and his students study the interaction of acoustic, ultrasonic, elastic, thermal, electromagnetic, and optical waves with materials, tissues, and structures. 